\title{
DEVELOPMENT AND EVALUATION OF A QUICK METHOD FOR OPTIMIZING A SPACE AND SIGNAL TIMING PLAN FOR ISOLATED SIGNALIZED INTERSECTIONS
}

\author{
Nedal RATROUT, Khaled ASSI* \\ Dept of Civil and Environmental Engineering, King Fahd University of Petroleum and Minerals, \\ Dhahran, Saudi Arabia
}

Submitted 16 December 2018; resubmitted 30 April 2019, 7 June 2019; accepted 18 July 2019;

first published online 18 May 2020

\begin{abstract}
One major cause of congestion at intersections is the fluctuation of traffic demand during the day. This phenomenon necessitates developing new models that can be used to enhance the performance of signalized intersections. We suggest a quick procedure for optimizing signal timing plans after identifying the best phasing scheme and selecting optimal lane allocation (space optimization) for any volume characteristics during the day at a typical four-leg intersection. The main contribution of this study is developing a method for collectively optimizing a signal-timing plan, intersection space, and phasing scheme. TRANSYT-7F, SYNCHRO and HCS2010 were used to assess the developed models in a case study. It was found that regardless of the optimization software used for timing-plan optimization, optimizing both space and timing plan together produce significant reductions in average intersection delay compared to optimizing only the timing plan. Furthermore, this study showed that the developed model, which optimizes space and time, consistently provided better results in terms of average intersection delay compared to TRANSYT-7F, SYNCHRO and HCS2010 in the case study.
\end{abstract}

Keywords: signalized intersection, pre-timed signal, space optimization, timing plan optimization, optimal phasing scheme, optimization model.

\section{Introduction}

The large variation in traffic demand at intersections during the day is a major contributor to urban congestion. Signal time optimization under a specific phasing scheme allocates signal timing to different traffic movements fairly under the constraints of fixed lane utilization. This study's main objective is to develop a quick and agile algorithm to find the optimal lane allocation (number of lanes for each movement), phasing scheme, cycle length, and green splits for a four-leg signalized intersection, which can be implemented quickly whenever volume characteristics change to ensure the most efficient signal operation all throughout the day. Signal phasing schemes at signalized intersections are usually selected based on the traffic demand characteristics. In approach-based phasing, each phase is fully protected and permits all movements (left, right, and through) of a given approach to move at the same time, as shown in Figure 1.

The primary concern of an approach-based phasing scheme at signalized intersections is the possibility of ca- pacity loss when there is significant variation in movement volumes served in the same phase. Lane usage violations in this phasing scheme (making turns from exclusive through lanes and vice versa) can also contribute to congestion. A movement-based phasing scheme, shown in Figure 2, serves traffic based on the movement type of opposing approaches and generally eliminates lane usage violations (Habibi 2016).

Nevertheless, the inefficiency of intersections operating in this phasing mode can still exist when there is a significant difference between the opposing traffic volumes of the same phase.

This paper suggests a quick procedure for optimizing the signal timing plan after identifying the best phasing scheme and selecting optimal lane allocation for any volume characteristics during the day at a typical four-leg intersection. From now onward, this process is referred to as optimizing the intersections' "time and space". The merit for this paper is the simplicity rather than the com-

*Corresponding author. E-mail: khaledassi@kfupm.edu.sa 


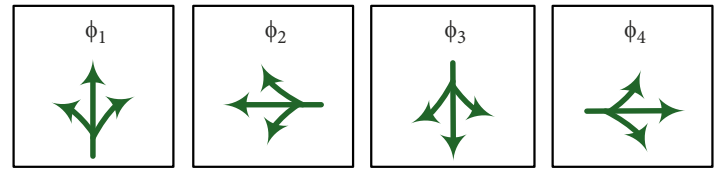

Figure 1. Approach-based phasing scheme

plexity and approximations rather than preciseness. Consequently, the findings lend themselves to be adaptable and programmable on smartphones and other personal devices. This is a useful merit in developing countries and in remote locations where a decent signal plan may be needed quickly for an isolated intersection. It should be mentioned that for testing purposes, the authors considered only the single ring controllers. Using a dual ring controller is a possible area for future research.

This study is presented in the following manner. Section 1 provides a brief overview of the literature related to space and time optimization for signalized intersections. Section 2 describes the model formulation. Section 3 presents a case study and the comparison of the developed models with other relevant software. Conclusions are available in the last section.

\section{Literature review}

The first optimal cycle length for 'mula was developed by Webster (1958) using an objective function of minimizing total delay for all vehicles. The proposed formula depends on the total lost time and summation of critical volume to saturation flow ratios (critical flow ratio), as shown in equation:

$$
C_{o p t}=\frac{1.5 \cdot L+5}{1-Y}
$$

where: $C_{o p t}$ is the optimal cycle length; $L$ is the total lost time; $Y$ is the summation of critical flow ratios.

Using Webster's formula, it can be observed that for the high value of critical flow ratio $Y$, the resultant cycle length will be either too large or the formula will be inapplicable when this value is equal to or exceeds one. Because of these major drawbacks, much research has been conducted to find the optimal cycle length, as shown in the following paragraphs.

Cheng et al. (2003) conducted a study to modify Webster's optimal cycle length equation by conducting experiments for a typical four-phase intersection with different scenarios of volume and lost time using Highway Capacity Manual (TRB 2010) delay equations. They developed three new models by recalibrating Webster's model, modifying Webster's model, and creating an exponential cycle length model. By comparing the optimal cycle length resulting from the developed models with that of Highway Capacity Software (HCS), they concluded that the developed models improved the accuracy of predicting the optimal cycle length. However, the recalibrated and modified Webster models were still inapplicable when the summation of critical flow ratios was equal to or exceeded one, as in the original Webster equation.

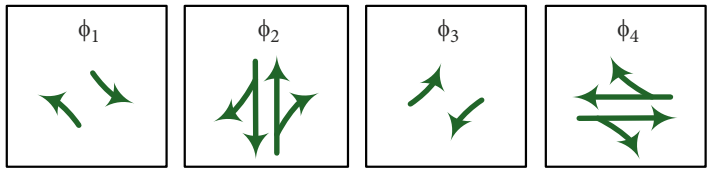

Figure 2. Protected movement-based signal phasing scheme

Zakariya and Rabia (2016) developed a new exponential regression model and calibrated Webster's formula by developing a search algorithm for finding the optimal cycle length that will minimize the intersection delay. They assumed the saturation flow rate is fixed for all movements at $1820 \mathrm{veh} / \mathrm{h}$. In addition, they used total lost times of 5, 6,7 and $8 \mathrm{~s}$ only. The main drawback of their developed model was the unrealistic cycle length predicted when the summation of critical flow ratios approached one. The calibrated and developed models were applicable only to the total lost times considered in the study.

Park and Kamarajugadda (2007) conducted a study to develop a genetic optimization algorithm model to optimize signal timing at signalized intersections considering the daily traffic demand fluctuation. They compared the results of the new model with the delay obtained from computerized software such as TRANSYT-7F and SYNCHRO. Based on the produced delay, it was revealed that signal timing plans generated from their genetic algorithm optimization model were superior.

Chin et al. (2011) developed a genetic algorithm model to optimize traffic signal timing, such as cycle length, green splits, and phasing sequences at multiple intersections with an objective function of minimizing delay. It was found that the new models had several advantages, such as the ability to accommodate the dynamic nature traffic networks condition by calibrating the system parameters accordingly.

He and Hou (2012) used the Ant Colony Algorithm (ACA) for traffic signal timing optimization using minimizing delay and the number of stops as objective functions. A comparison between ACA and the other traditional techniques, such as genetic algorithm and the Webster algorithm, was conducted. The comparison results revealed that ACA was superior regarding minimizing delays and number of stops.

Many studies were conducted to compare between different signal timing optimization software. Portugais (2013) compared the optimized signal timing resulting from TRANSYT-7F and VISTRO based on different performance measures, such as Level Of Service (LOS), control delay, and queue lengths. Based on these performance measures, it was concluded that TRANSYT-7F outperformed VISTRO.

Benekohal et al. (2002) conducted a study to compare between HCS, SYNCHRO and PASSER. The comparison was based mainly on the amount of the delay reduction materialized when optimized signal settings were implemented. CORSIM was used to assess the impacts of optimization. CORSIM simulation results revealed that, for Synchro, the delays for optimized conditions were significantly lower than the delays before optimization. 
Most signal control strategies worldwide assume fixed lane utilization at intersection approaches. This is called Fixed Lane Assignment (FLA) and it might degrade the intersection's performance under fluctuating demand characteristics throughout the day. Dynamic Lane Assignment (DLA) is an Intelligent Transportation System (ITS) application in which the lane allocation depends mainly on real-time turning movement demand. Optimizing the space at signalized intersections in urban areas was considered in many studies worldwide. Zhong et al. (2008) analyzed the impacts of DLA at one approach of a signalized intersection. A model for optimizing a time-space combination was proposed. The analysis results revealed the proposed method produces the optimum benefit scheme.

Wu et al. (2012) used PARAMICS simulation software to analyse the effects of DLA on one approach of a hypothetical isolated signalized intersection with predefined demand levels. It was revealed the DLA strategy enhances the operational performance regarding a reduction in average vehicle delay and the number of stops. Zhang and $\mathrm{Wu}$ (2012) analysed the effects of dynamic lane grouping using mathematical programming at a hypothetical isolated signalized intersection assuming predefined demand levels and a fixed cycle length of $120 \mathrm{~s}$. In the analysed scenario, only one approach had variable traffic demand and DLA. The objective function was minimizing the maximum flow ratio. It was concluded the lane-grouping strategy improves the mobility performance in terms of reduced average vehicle delay and the number of stops.

Zhao et al. (2013a, 2013b) built an optimization model for DLA using integer nonlinear programming. The objective function was to minimize the sum of the critical flow ratios. Two cases were considered in their study. In the first case, they applied the model to one approach only and, in the second case, they applied it to two opposing approaches only. Compared to fixed lane allocation, the intersection delay decreased by $14.70 \%$ after applying the new optimization model. However, they did not apply their models to approaches simultaneously.

Alhajyaseen et al. (2017a, 2017b) investigated the effectiveness of optimizing the space accompanied with signal timing optimization on the performance of signalized intersections. An optimization model was developed using the MATLAB environment with an objective function of minimizing average intersection delay. It was concluded that optimizing the space along with signal timing optimization could enhance the signalized intersection's performance. However, they considered an approach-based phasing scheme only and assumed shared lanes only exist on the far left and right lanes.

Assi and Ratrout (2018) developed a new quick method to find the optimal lane grouping for three and fourlane approaches at isolated signalized intersections for any combination of turning movement. A MATLAB optimization model was developed to find the optimal lane assignment for a massive turning movement combination using an objective function of minimization of average intersection delay. It was found that the percentage of turning movements at an approach is sufficient to identify the optimal lane grouping at that approach.

Zhao et al. (2017) attempted to evaluate the efficiency of applying DLA at signalized intersections using field data. In this study, the DLA strategy was applied at one approach of five signalized intersections in Shanghai (China). Based on the operational efficiency analysis, it was found that applying DLA decreased the saturation flow rate significantly.

Yao et al. (2018) studied optimizing signal phasing plans, signal timing, and left-lane length for isolated signalized intersections. They developed two optimization models using an objective function for minimizing intersection delays for the first model and maximizing the ratio of intersection capacity to intersection delay for the second model. They assumed fixed allocation of lanes at all approaches with two lanes for left-turning movement, one lane for through movement, and one lane shared between through and right-turning movements. A microscopic simulation tool (VISSIM) was used in this study to evaluate the intersection's performance under different signal phase sequences. It was found that the signalphasing sequence had a significant effect on intersection performance.

The authors could not find any study for collectively optimizing the signal timing, intersection space (lane allocation) and phasing scheme. Hence, this study aims to fill this gap and provide researchers and engineers with a simple method that can be quickly used to determine the optimal phasing scheme, optimal allocation of lanes, and optimal signal timing plan for any typical four-leg isolated signalized intersection. The proposed method was compared with the available commercial signal timing optimization tools, such as HCS, SYNCHRO and TRANSYT-7F.

\section{Model formatting}

This study is aimed at optimizing the space and time at a typical four-leg signalized intersection by first finding the optimal number of lanes needed for each movement at each approach and selecting the optimal phasing scheme. Following that, the optimal cycle length is then determined and its green time is allocated proportionally to the traffic volume of different phases using the critical movement analysis (TRB 2010).

Two optimization processes are developed first: space optimization followed by signal-timing optimization. For space optimization, a specific optimization model was developed for approach-based phasing and another for movement-based phasing using the following two assumptions:

"» all left turns are fully protected (requirement from local authorities to enhance safety);

"» right turns on red are prohibited at the intersection; 
"m) the principle of equal saturation flow ratio is used for shared lanes; according to this principle, the traffic demand is distributed between the lanes serving the same movement in such a way to keep the volumeto-saturation flow ratios for these lanes nearly equal. It should be mentioned that, in developing the optimization model, each of the opposing approaches (i.e., east-west or north-south) is considered separately. Considering the opposing approaches rather than the whole intersection is more flexible and allows two opposing approaches to operate under one phasing scheme while the other approaches can operate with the second phasing scheme, if needed. Using a mix of both phasing schemes at the same intersection might be more efficient in certain circumstances.

The space optimization process starts by determining the optimal number of lanes per movement using an objective function of minimizing the difference between the volumes per lane over all lanes that will be served in a specific phase. This will have a significant effect on minimizing the unused green time of specific movement during a phase serving more than one movement or more than one approach. This optimal number of lanes depends primarily on the volume characteristics and phasing scheme used. Consequently, to find the optimum answer, each phasing scheme should be examined independently.

\subsection{Model formulation for an approach-based phasing scheme}

The opposing approaches operate independently under the approach-phasing scheme (as shown in Figure 1). Hence, the optimization model for an approach-based phasing scheme should be applied to each approach separately. The objective function and the constraints for an approach-based optimization model are shown in the following formulation:

$\min \left(\left(\frac{V_{a, 1}}{N_{a, 1}}-\frac{V_{a, 2}}{N_{a, 2}}\right)+\left(\frac{V_{a, 1}}{N_{a, 1}}-\frac{V_{a, 3}}{N_{a, 3}}\right)+\left(\frac{V_{a, 2}}{N_{a, 2}}-\frac{V_{a, 3}}{N_{a, 3}}\right)\right)$

subject to:

$N_{a, j}>0$;

$\sum_{j=1}^{3} N_{a, j}=N_{a}$

where: $j$ - turning movement at the approach $(j=1,2,3$, respectively representing left-turn movement, through movement, and right-turn movement); $a$ - intersection approach ( $a=1,2,3,4$, respectively representing west approach, north approach, east approach and south approach); $N_{a}$ - total number of lanes at approach $a ; N_{a, j}-$ number of lanes for turning movement $j$ at approach $a$; $V_{a, j}$ - traffic volume of movement $j$ at approach $a$.

\subsection{Model formulation for a movement-based phasing scheme}

In this kind of phasing scheme, any two opposing approaches operate together as one unit and are usually served in two phases. Applying a movement-based phasing scheme prohibits sharing left-turning movement with through movement. Because of that, the number of lanes for left-turning movement is forced to be an integer, which means only exclusive left-turning lanes can be used to serve left-turning traffic (i.e., a shared lane for left movement and through movement is not allowed). Again, as the right of way for opposing approaches is concurrent, the following model should be applied on a direction-wise (east-west or north-south) basis:

$\min \left(\left(\frac{V_{a, 1}}{N_{a, 1}}-\frac{V_{a+2,1}}{N_{a+2,1}}\right)+\left(\frac{V_{a, 2}+V_{a, 3}}{N_{a, 2}+N_{a, 3}}-\frac{V_{a+2,2}+V_{a+2,3}}{N_{a+2,2}+N_{a+2,3}}\right)\right)$

subject to:

$N_{a, j}>0$;

$N_{a, 1}, N_{a+2,1}$ are integers;

$N_{a, 1}, N_{a+2,1}, N_{a, 2}, N_{a, 3}, N_{a+2,2}, N_{a+2,3}<N_{a}$;

$\sum_{j=1}^{3} N_{a, j}=N_{a}$;

$\sum_{j=1}^{3} N_{a+2, j}=N_{a+2}$.

For simplicity and consistency, all volumes mentioned in the objective functions (Equations (2) and (3)) are the equivalent through traffic volumes. The turning traffic volumes are converted to the equivalent through traffic volumes using the through equivalent factor, which is the ratio between the saturation flow rate of through movement to the saturation flow rate of the turning movement. The saturation flow rates for turning movements are determined using equation (Zhang, Wu 2012; Kimber et al. 1986):

$$
S_{a, k}=\frac{\bar{S}_{a, k}}{1+1.5 \cdot \sum_{j=1}^{j=3} \frac{f_{a, k, j}}{r_{a, k, j}}},
$$

where: $S_{a, k}$ - saturation flow rate of lane $k$ at approach $a$; $\bar{S}_{a, k}$ - saturation flow rate for straight movement (assumed to be $1900 \mathrm{veh} / \mathrm{h}$ ) (Zhang, Wu 2012; Kimber et al. 1986); $r_{a, k, j}$ - turning radius for movement $j(=\infty$ for straightahead movement); $f_{a, k, j}$ - flow factor (the proportion of movement $j$ at lane $k$ of approach $a$ from total traffic at lane $k$, as shown in equation):

$$
f_{a, k, j}=\frac{V_{a, k, j}}{\sum_{j=1}^{j=3} V_{a, k, j}}
$$


where: $V_{a, k, j}$ is the traffic demand of movement $j$ via lane $k$ at approach $a$.

Applying the above equation on a typical four-leg intersection in the study area resulted in saturation flow rates for left-turning movement and right-turning movement of 1690 and $1652 \mathrm{veh} / \mathrm{h}$, respectively. This corresponds to equivalent through factors for left and rightturning movements of 1.12 and 1.15 , respectively.

Using the optimal number of lanes found for each phasing scheme (Equations (2) and (3)), the flow ratio is calculated for all lanes for each phasing scheme. The flow ratio for a lane is defined as the ratio of traffic volume $v$ on the lane to its saturation flow rate $s$. Then, the critical flow ratio for each phase $\left(\frac{v}{s}\right)_{\text {critical, } i}$ is determined. The critical flow ratio for a phase is the maximum lane's flow ratio served in that phase. For example, for a four-phase signalized intersection, there are four critical flow ratios (one for each phase). The phasing scheme resulting in the minimum summation of critical flow ratios is selected as the optimal phasing scheme. The summation of critical flow ratios $Y$ can be found using equation:

$$
Y=\sum_{i=1}^{N}\left(\frac{v}{s}\right)_{c r i t i c a l, i},
$$

where: $i$ is the phase number; $N$ is the number of phases.

The next step after finding the optimal phasing scheme and lane allocation is to find the optimal cycle length for the intersection.

Identifying the optimal cycle length is a lengthy iterative process of testing all possible cycles within a specific range and then determining the cycle length that satisfies a specific objective function. In this study, the objective function of cycle length optimization is minimizing the average intersection delay. To estimate average intersection delay per vehicle, the proposed methodology by the Highway Capacity Manual (TRB 2010) is used. The average control delay per vehicle for a given lane is given by equation:

$$
d_{a, k}=d_{1, a, k} \cdot P F+d_{2, a, k}+d_{3, a, k}
$$

where: $d_{a, k}$ - control delay per vehicle $[\mathrm{s}] ; d_{1, a, k}-$ uniform control delay assuming uniform arrivals for lane $k$ of approach $a$ [s]; $P F$ - progression adjustment factor (assumed to be 1$) ; d_{2, a, k}$ - average delay per vehicle owing to random arrivals for lane $k$ of approach $a$, which is called incremental delay $[s] ; d_{3, a, k}$ - average delay per vehicle owing to an initial queue at the start of the analysis period for lane $k$ of approach $a$ [s].

The average delay for lane $k$ at approach $a$ owing to uniform arrivals is estimated using Equation (8) (TRB 2010). The progression adjustment factor $P F$ is assumed to be 1 , as we are considering isolated intersections only (TRB 2010):

$$
d_{1, a, k}=\frac{0.5 \cdot C \cdot\left(1-\frac{g_{i}}{C}\right)^{2}}{1-\left(\min \left(1, x_{a, k}\right) \cdot \frac{g_{i}}{C}\right)},
$$

where: $C$ - cycle length $[\mathrm{s}] ; g_{i}$ - effective green time for lane group $[\mathrm{s}] ; x_{i, k}$ - total lane volume-to-capacity ratio $v / c$ for lane $k$, where $c_{i, k}=S_{i, k}\left(\frac{g_{i}}{C}\right), c_{i, k}$ is the capacity of lane $k[\mathrm{veh} / \mathrm{h}] ; S_{i, k}$ is the saturation flow rate for lane $k[\mathrm{veh} / \mathrm{h}]$.

The incremental delay $d_{2}$ is estimated following equation (TRB 2010):

$$
\begin{aligned}
& d_{2, a, k}=900 \cdot T \times \\
& \left(\left(x_{a, k}-1\right)+\sqrt{\left(x_{a, k}-1\right)^{2}+\frac{8 \cdot k_{f} \cdot I \cdot x_{a, k}}{c_{a, k} \cdot T}}\right),
\end{aligned}
$$

where: $T$ - duration of the analysis period $[\mathrm{h}] ; k_{f}-$ incremental delay factor; $I$ - upstream filtering/metering adjustment factor; $c_{a, k}$ - lane capacity [veh/h].

As the model is developed for an isolated signalized intersection, the value of the upstream filtering-metering adjustment factor $I$ is assumed as 1.0 while the value of the incremental delay factor $k_{f}$ is assumed as 0.5 because the signal operation is not actuated as recommended by Highway Capacity Manaual (TRB 2010). For simplification, it is assumed there is no initial queue delay from the previous analysis period, which means $d_{3}$ is 0 (Cheng et al. 2003; Ding et al. 2014).

Using MATLAB, a brute force hill-climbing (exhaustive search) algorithm was developed to find the optimal cycle length for any traffic volume combinations. This algorithm systematically enumerates all possible cycle lengths within a specific range then picks the cycle length that results in minimum average intersection delay. In this study, it was assumed the maximum cycle length is $300 \mathrm{~s}$, which is already the maximum cycle length at some congested intersections in the study area. It should be mentioned that the minimum cycle length required to serve pedestrians is $60 \mathrm{~s}$ (TRB 2010). However, considering the minimum lost time per phase as a limiting boundary for cycle length determination, an absolute theoretical minimum cycle of $10 \mathrm{~s}$ is taken as comprehensive from a research and curiosity perspective in this study. Consequently, the investigated cycle range was from 10 to $300 \mathrm{~s}$. The green time for each phase (split) was calculated using the time budget concept in which the total effective green time is distributed between the phases based on the critical flow ratio for each phase (Roess et al. 2010). The relationship between the optimal cycle length and the summation of critical flow ratios $Y$ (Equation (6)) was investigated for different values of $Y$ between 0.1 and 1.10 and with eight values of total lost time $L$ for each value of $Y$ investigated. Total lost time is defined as the summation of startup lost time and clearance lost time (Roess et al. 2010). 
For each value of $Y, 20$ different hypothetical turning volume combinations of all approaches (which may result in different total intersection volume) that produce the targeted $Y$ were generated randomly. All cycle lengths in the investigated range $(10 \ldots 300 \mathrm{~s})$ were tried on each of the 20 generated traffic volume combinations. Then, the optimal cycle length for each combination resulting in the minimum average intersection delay was recorded. It was found that the maximum difference between the 20 values of cycle lengths for the same $Y$ was less than $10 \mathrm{~s}$ despite the total intersection volume and its turning movement's characteristics, which were different for each trial. Thus, it seems the optimal cycle length depends mainly on the value of $Y$ regardless of the absolute volume and turning movements producing this $Y$. Hence, the optimal cycle length for any value of $Y$ was taken as the average of the twenty cycle lengths. The Figures 3 and 4 represent the relationship between the optimal cycle length and summation of critical flow ratios for different values of total lost time ranging between 6 and $20 \mathrm{~s}$. The authors considered
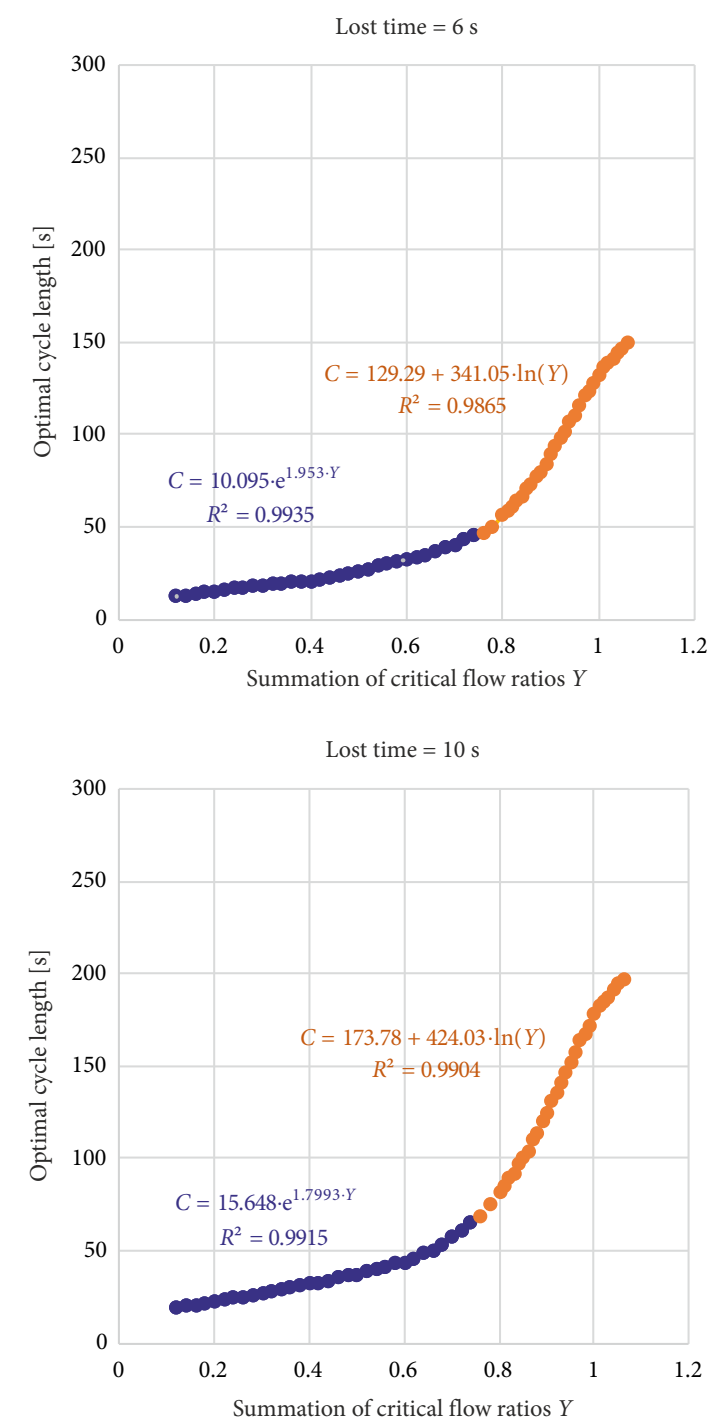

this wide range of lost time to ensure the study's outputs will be applicable for most practical situations.

It can be observed from Figures 3 and 4 that the relationship between the optimal cycle length and $Y$ starts changing from exponential to logarithmic around $Y=$ 0.74 . Hence, it was decided to develop two different equations (exponential and logarithmic) to describe the relationship between the optimal cycle length and $Y$ for each value of total lost time. The exponential equations were developed to be used when $Y \leq 0.74$, while the logarithmic equations can be used for $Y>0.74$. Moreover, it is clear from the figures above that the values of coefficients of determination $R^{2}$ are high for all equations. This indicates most of the variation in the optimal cycle length can be explained using $Y$. The authors attempted to combine all of the above equations into two equations only (exponential equation for $Y \leq 0.74$ and logarithmic equation for $Y>0.74$ ) by considering the total lost time as an independent variable. However, this attempt resulted in degradation in the prediction capability of the optimal cycle length.
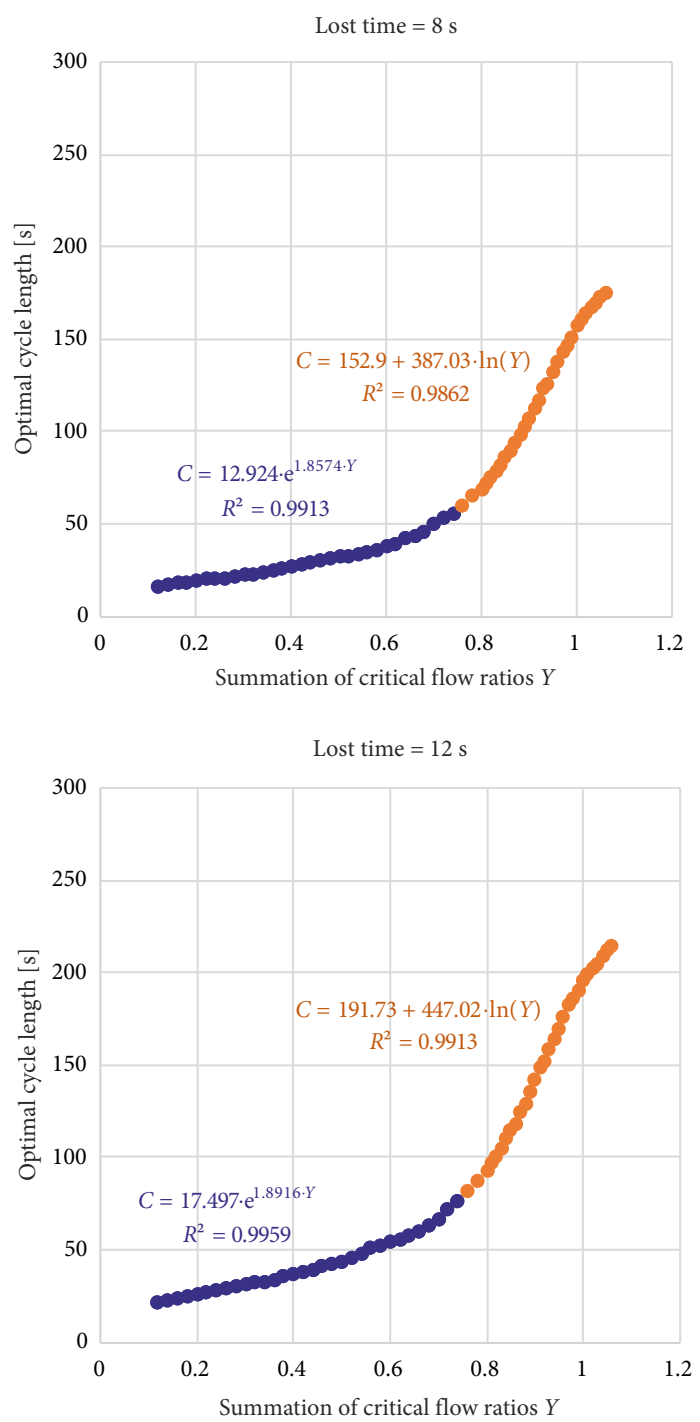

Figure 3. Optimal cycle length at different values of $Y$ for lost times between 6 and $12 \mathrm{~s}$ 
This degradation was more significant in logarithmic equations.

The developed regression models for predicting optimal cycle length were tested on a hypothetical traffic data set, which has not been used in developing the models. A data set of 300 turning volume combinations was generated randomly. For each turning volume combination, the optimal cycle length was found using the brute force hillclimbing algorithm in MATLAB. This "true" optimal cycle length was then compared to the estimated optimal cycle length using the developed regression models (Figures 3 and 4). The error was calculated by dividing the difference between the "true" optimal cycle length and estimated optimal cycle length by the "true" optimal cycle length.

It was found that the percentage error ranges from 0 to $7.6 \%$ with an average of $2.2 \%$ and a standard deviation of $1.6 \%$. Based on percentage error results, it can be concluded that using the developed regression model for optimal cycle length prediction can produce a reasonable optimal cycle length quickly compared to the hill-climb-
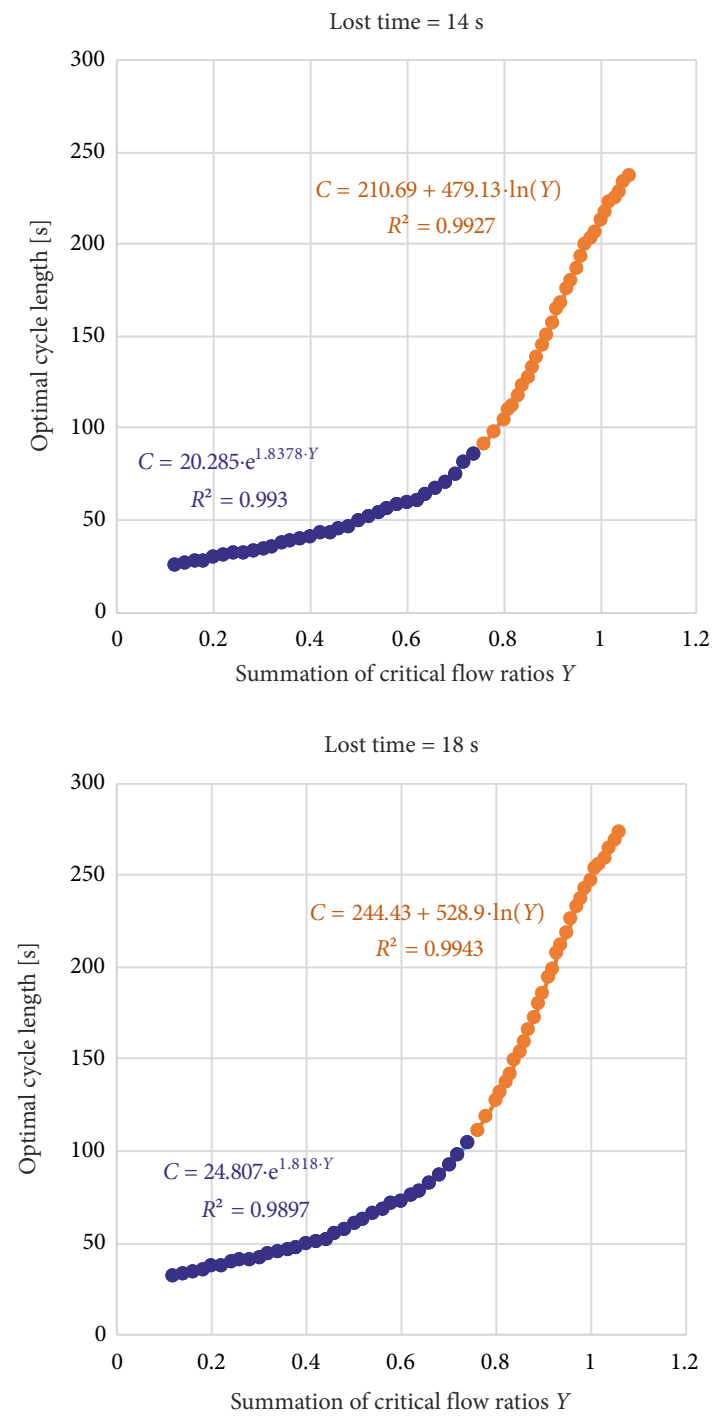

ing algorithm, which needs more execution time. Figure 5 represents the flowchart of the proposed models for selecting an optimum phasing scheme for any opposing approaches. The optimal cycle can be either determined by the proposed quick method (i.e., Figures 3 and 4) or by using any commercial software, as shown at the end of Figure 5. Green splits can be estimated using the proposed method by distributing the cycle length between approaches based on their proportional volumes. Commercial software produce cycle length together with green splits and there is no need for manual intervention.

\section{Case study}

The developed models were assessed using a simulation environment (commercial signal simulation programs) on field data of a typical four-leg isolated signalized intersection. The chosen intersection is subjected to tidal flow during the day and operates under an approach-based phasing scheme using four-phases (one phase for each approach).
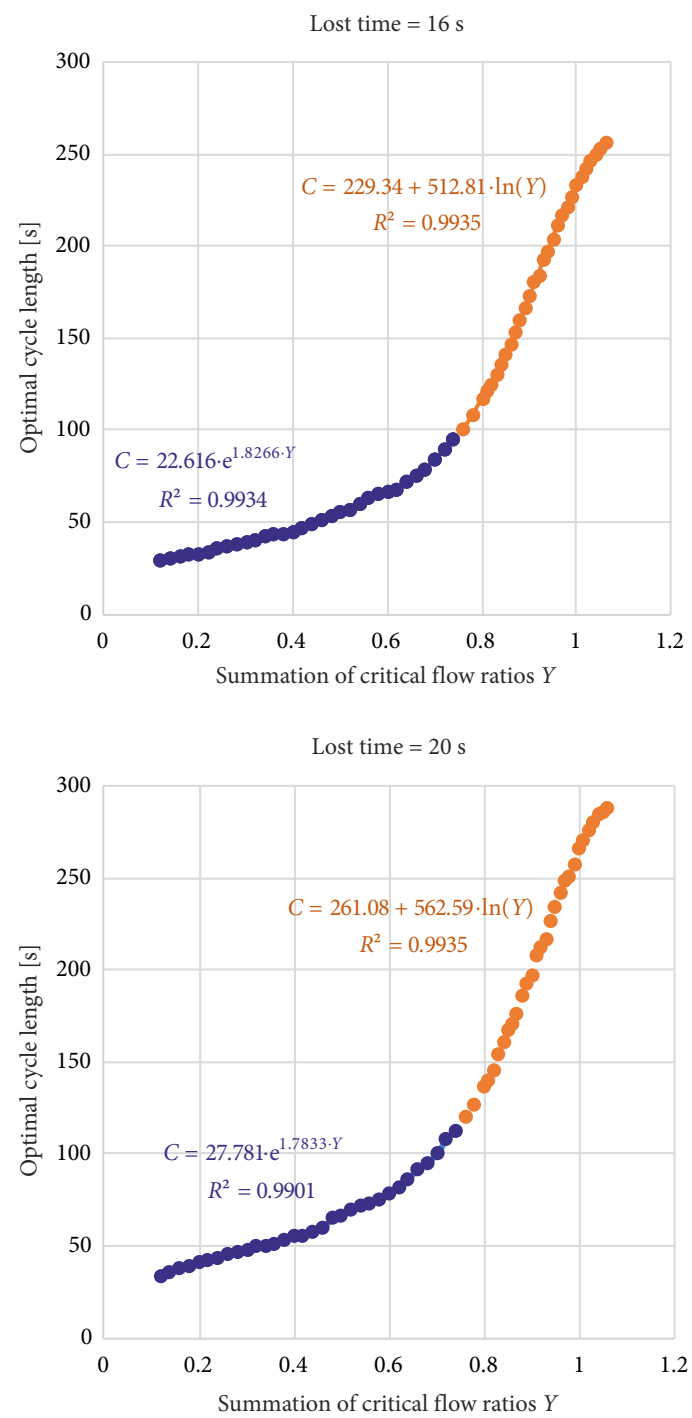

Figure 4. Optimal cycle length at different values of $Y$ for lost times between 14 and $20 \mathrm{~s}$ 


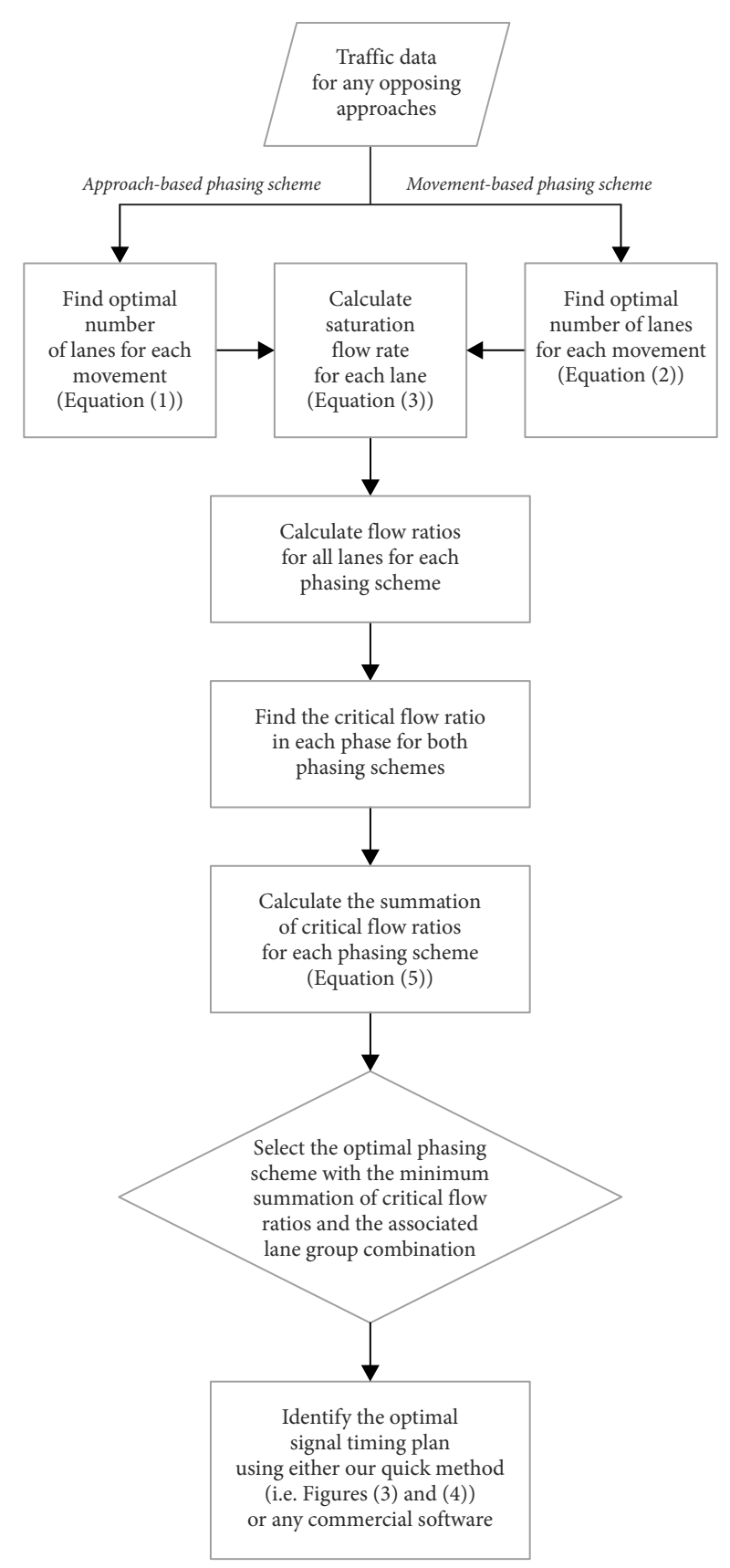

Figure 5. Flowchart of the proposed model

Hourly turning movement volumes (left, through and right) were collected at all approaches of the intersection for 24 consecutive hours on a typical weekday. The chosen intersection has five peak hours during the day (two morning peak hours, one afternoon peak hour, and two evening peak hours). The simulation analysis was restricted to these five hours for convenience. The hourly volumes during these periods are given in Table 1. Currently, the intersection operates with one lane utilization plan for all of the five peak hours, as shown in the top left corner of Figure 6.

The performance of the developed space optimization model was evaluated over the five peak hours based on average intersection delay. The objective was to evaluate the effectiveness of using the developed model in optimizing the space, specifically phasing scheme and lane uses (i.e., the number of lanes allocated for each movement in all phases). For this purpose, three macroscopic optimization software (TRANSYT-7F, SYNCHRO and HCS2010) were used. TRANSYT-7F and SYNCHRO models are the only models known to be calibrated for the study area. The Highway Capacity Manual (TRB 2010) and its associated software HCS2010 are well-known and acceptable standard tools in the study area for traffic signal design and analysis.

The first step in the evaluation process was developing an optimal signal-timing plan using TRANSYT-7F with the existing phasing scheme and lane utilization. Following that, the developed model for space optimization was used to identify the optimum phasing scheme and lane utilization for each peak period. Figure 6 represents the existing and optimal lane utilization and the optimal lane utilization for each peak hour. TRANSYT-7F was then used to obtain the optimal timing plan for the optimum lane utilization. The resulting delay of this signal plan was compared with that of the first plan to gauge the benefits of space optimization. The results are summarized in Table 1, which shows there is considerable benefit from applying the optimal space scheme with the optimal time plan. The reduction of delay from such effort ranged from 78 to $92 \%$ with an average of $87 \%$, as shown in the last column of Table 1 . The same comparison procedure was repeated using HCS2010, which resulted in a reduction between 68 and $88 \%$ with an average of $81 \%$. Repeating this comparison using Synchro, produced a reduction ranging between 54 and $70 \%$ with an average of $64 \%$. This indicates that regardless of the optimization tool used for optimizing the timing plan, optimizing space (using the developed space optimization algorithm) together with the timing plan always yield significant reductions in average intersection delay compared to optimizing the timing plan only. This indicates that optimizing the space through the developed models is as important as optimizing the signal timing plan. Consequently, the developed space optimization algorithm can be a valuable add-on algorithm to the commercial time optimization programs used in this paper.

Table 2 shows performance comparisons between the proposed complete model (capable of optimizing space utilization, phasing scheme, and signal timing) and the available commercial tools that optimize timing only. The comparisons were based on the average intersection delay resulting from SIMTRAFFIC, which is a microscopic simulation tool. SIMTRAFFIC was used as a common simulation yardstick between the developed model and other commercial programs for fairness and objectivity. It is clear from Table 2 that the developed optimal solution is superior in terms of average intersection delay compared to the optimal plan produced by TRANSYT-7F. The reduction in delay was between 48 and $86 \%$ with an average of $71 \%$. 

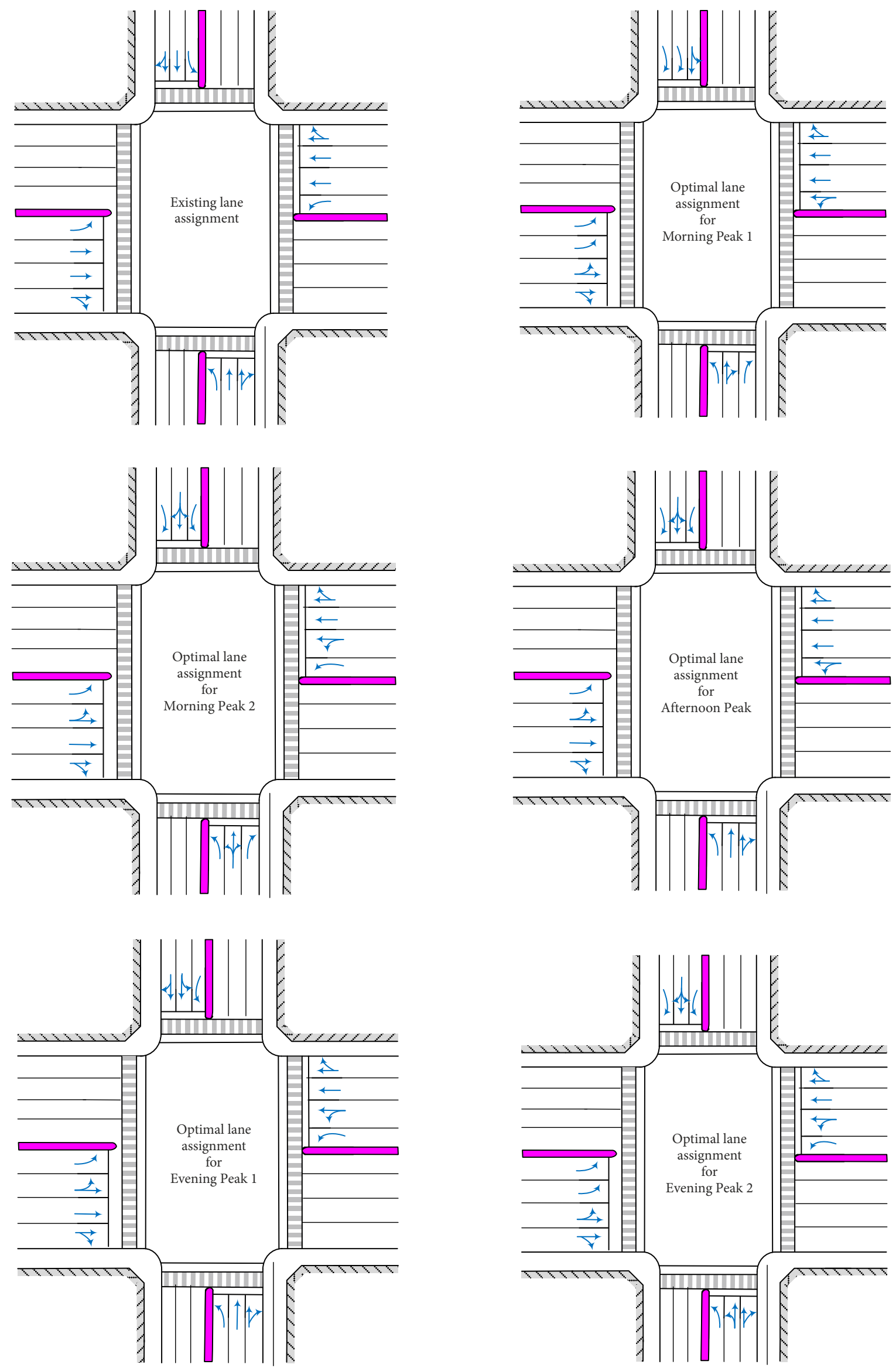

Figure 6. Existing and optimal lane assignment for the studied intersection in each peak hour 
Table 1. TRANSYT-7F average intersection delay with the optimal TRANSYT-7F timing plan and the effect of the space optimization

\begin{tabular}{|l|c|c|c|}
\hline $\begin{array}{c}\text { Peak hour } \\
\text { (total intersection volume } \\
\text { [veh/h]) }\end{array}$ & $\begin{array}{c}\text { Optimal TRANSYT-7F timing plan } \\
\text { with existing lane allocation [s/veh] } \\
\text { (optimal cycle length [s]) }\end{array}$ & $\begin{array}{c}\text { Developed optimal space utilization with } \\
\text { optimal TRANSYT-7F timing plan } \\
\text { [s/veh] }]^{*} \text { (optimal cycle length [s]) }\end{array}$ & $\begin{array}{c}\text { Reduction } \\
\text { in delay [\%] }\end{array}$ \\
\hline Morning Peak 1 (3547) & $281.50(202)$ & $23.50(47)$ & 92 \\
\hline Morning Peak 2 (3641) & $103.40(158)$ & $23.0(43)$ & 78 \\
\hline Afternoon Peak (4494) & $348.90(210)$ & $42.40(80)$ & 88 \\
\hline Evening Peak 1 (4991) & $373.40(196)$ & $31.90(95)$ & 91 \\
\hline Evening Peak 2 (4506) & $407.80(168)$ & $47.70(84)$ & 88 \\
\hline Average & & & 87 \\
\hline
\end{tabular}

Note: ${ }^{*}$ - optimization of space was conducted using the developed mode.

Table 2. SIMTRAFFIC simulation of average intersection delay for the proposed process and TRANSYT-7F

\begin{tabular}{|l|c|c|c|}
\hline \multicolumn{1}{|c|}{ Peak hour } & $\begin{array}{c}\text { Optimal TRANSYT-7F timing plan } \\
\text { with existing lane allocation [s/veh] } \\
\text { (optimal cycle length [s]) }\end{array}$ & $\begin{array}{c}\text { Developed optimal space utilization with } \\
\text { developed optimal timing plan [s/veh] } \\
\text { (optimal cycle length [s]) }\end{array}$ & $\begin{array}{c}\text { Reduction } \\
\text { in delay [\%] }\end{array}$ \\
\hline Morning Peak 1 & $122.50(202)$ & $49.3(52)$ & 60 \\
\hline Morning Peak 2 & $58.60(158)$ & $30.4(51)$ & 48 \\
\hline Afternoon Peak & $254.20(210)$ & $50.20(79)$ & 80 \\
\hline Evening Peak 1 & $371.20(196)$ & $53.80(92)$ & 86 \\
\hline Evening Peak 2 & $272.70(168)$ & $46.50(84)$ & 83 \\
\hline Average & & & 71 \\
\hline
\end{tabular}

Redoing the comparison using HCS2010 produced a reduction in delay between 53 and $83 \%$ with an average of $75 \%$. SYNCHRO was also tried and produced similar results, namely, the delay reduction ranged between 35 and $84 \%$ with an average of $67 \%$. Consequently, the proposed model for space and time optimization consistently provided better results regarding average intersection delay compared to all the commercial models we tested.

\section{Conclusions}

In this study, we developed an optimization model to optimize the space (phasing scheme and lane allocation) and timing plan for signalized intersections. The proposed model starts with selecting an optimal phasing scheme with lane utilization, determining optimal cycle length, and, finally suggesting best green time splits. Two phasing schemes were considered: the approach-based phasing scheme and the movement-based phasing scheme. The phasing scheme that results in the minimum summation of critical flow ratios was selected as the optimal phasing scheme for a specific turning volume combination. The critical flow ratio for a phase is defined as the maximum lane flow ratio served in that phase. The objective function of the timing plan optimization model was to minimize the average intersection delay following the Highway Capacity Manual (TRB 2010) procedure for delay calculations. Two equations (exponential and logarithmic) were developed to describe the relationship between the optimal cycle length and the summation of critical flow ratios $Y$ for eight values of total lost time. The exponential equations were developed for use when $Y \leq 0.74$, while the logarithmic equations can be used for $Y>0.74$. The developed regression models for predicting optimal cycle length were tested on a hypothetical data set of 300 turning volume combinations. For each turning volume combination, the optimal cycle length was found using the brute force hill-climbing algorithm in MATLAB based on minimum average intersection delay. This "true" optimal cycle length was then compared to the estimated optimal cycle length using the developed regression models. Based on the comparative analysis results, it was found the percentage error ranges from 0 to $12.4 \%$ with an average of $2.4 \%$.

Three macroscopic signal optimization programs, namely, TRANSYT-7F, HCS2010 and SYNCHRO were used to assess the developed optimization model on a typical four-leg isolated signalized intersection. All the programs optimize timing plans without considering lane utilization and phasing scheme, per se. The optimization programs TRANSYT-7F, HCS2010 and SYNCHRO were used to find the optimal timing plans before and after optimizing the space with our developed space optimization algorithm. The average intersection delay resulting from optimizing the signal timing only was compared with that resulting from optimizing the space and signal timing together. It was found that regardless of the optimization program used for timing-plan optimization, optimizing space and timing plan together always yields significant reductions in average intersection delay compared to opti- 
mizing the timing plan only. This study's findings are supported by the findings of previous studies, which found that optimizing phasing scheme, space, and timing plan have a significant effect on enhancing the traffic operations of signalized intersections. However, no researcher attempted to optimize phasing scheme, signal timing, and space altogether. Further, this study showed that the developed model, which optimizes space and time, consistently provided better results regarding average intersection delay compared to the three commercial optimization programs tested in this paper. Ultimately, this study provides a quick and efficient procedure for holistically optimizing the signal-timing plan, intersection space, and phasing scheme. This can be helpful in DLA and pre-signal applications. For future work, the authors will try to evolve the developed model to incorporate more sophisticated phasing schemes using the principles of leading/lagging green, multiple rings design, and phase skipping to achieve more efficient use of green times and, consequently, minimize intersection delay further. Moreover, the authors will look to incorporate additional left-turn phasing options, such as permissive and protected-permissive phasing. The authors will also try to program the developed procedure on smartphones through developing applications for IOS and Android systems.

\section{Acknowledgements}

The authors would like to acknowledge the support provided by the Deanship of Scientific Research at King Fahd University of Petroleum and Minerals (KFUPM), Dhahran (Saudi Arabia).

\section{Author contributions}

The authors confirm contribution to the paper as follows:

"'" study conception and design: Nedal Ratrout;

"») data collection: Nedal Ratrout, Khaled Assi;

"» analysis and interpretation of results: Nedal Ratrout, Khaled Assi;

"» draft manuscript preparation: Khaled Assi, Nedal Ratrout.

All authors reviewed the results and approved the final version of the manuscript.

\section{References}

Alhajyaseen, W. K. M.; Najjar, M.; Ratrout, N. T.; Assi, K. 2017a. The effectiveness of applying dynamic lane assignment at all approaches of signalized intersection, Case Studies on Transport Policy 5(2): 224-232.

https://doi.org/10.1016/j.cstp.2017.01.008

Alhajyaseen, W. K.; Ratrout, N. T.; Assi, K. J.; Hassan, A. A. $2017 \mathrm{~b}$. The integration of dynamic lane grouping technique and signal timing optimization for improving the mobility of isolated intersections, Arabian Journal for Science and Engineering 42(3): 1013-1024.

https://doi.org/10.1007/s13369-016-2274-4
Assi, K. J.; Ratrout, N. T. 2018. Proposed quick method for applying dynamic lane assignment at signalized intersections, IATSS Research 42(1): 1-7.

https://doi.org/10.1016/j.iatssr.2017.03.004

Benekohal, R. F.; Elzohairy, Y. M.; Saak, J. E. 2002. Comparison of delays from highway capacity software, SYNCHRO, PASSER II and IV, and CORSIM for urban arterials, Transportation Research Record: Journal of the Transportation Research Board 1802: 133-144. https://doi.org/10.3141/1802-16

Cheng, D.; Messer, C. J.; Tian, Z. Z.; Liu, J. 2003. Modification of Webster's minimum delay cycle length equation based on HCM 2000, in Transportation Research Board 82nd Annual Meeting Compendium of Papers CD-ROM, 12-16 January 2003, Washington, DC, US, 1-27.

Chin, Y. K.; Yong, K. C.; Bolong, N.; Yang, S. S.; Teo, K. T. K. 2011. Multiple intersections traffic signal timing optimization with genetic algorithm, in 2011 IEEE International Conference on Control System, Computing and Engineering, Penang, Malaysia, 454-459. https://doi.org/10.1109/ICCSCE.2011.6190569

Ding, J.; Zhou, H.; Yao, R. 2014. Optimization of lane use and signal timing for isolated signalized intersections with variable lanes, in J. Ma, Y. Yin, H. Huang, D. Pan (Eds.). CICTP 2014: Safe, Smart, and Sustainable Multimodal Transportation Systems, 2012-2024. https://doi.org/10.1061/9780784413623.193

Habibi, H. 2016. Assessment of Dynamic Lane Grouping for Isolated Signalized Intersection and Application of Machine Learning Models. MSc Thesis. King Fahd University of Petroleum and Minerals, Dhahran, Saudi Arabia. 132 p. Available from Internet: https://eprints.kfupm.edu.sa/id/eprint/140052

He, J.; Hou, Z. 2012. Ant colony algorithm for traffic signal timing optimization, Advances in Engineering Software 43(1): 14-18. https://doi.org/10.1016/j.advengsoft.2011.09.002

Kimber, R. M.; McDonald, M.; Hounsell, N. B. 1986. The Prediction of Saturation Flows for Road Junctions Controlled by Traffic Signals. Transport and Road Research Laboratory (TRRL) Research Report 67. Crowthorne, Berkshire, UK. 21 p. Available from Internet: https://rl.co.uk/reports/RR67

Park, B.; Kamarajugadda, A. 2007. Development and evaluation of a stochastic traffic signal optimization method, International Journal of Sustainable Transportation 1(3): 193-207. https://doi.org/10.1080/15568310600737568

Portugais, B. 2013. A Comparison of Signal Optimization Results on the Eagle Road Corridor in Meridian, Idaho Using TRANSYT-7F and VISTRO. Technical report. Boise State University, US. 39 p. https://doi.org/10.13140/2.1.2206.3680

Roess, R. P.; Prassas, E. S.; McShane, W. R. 2010. Traffic Engineering. Pearson. $752 \mathrm{p}$.

TRB. 2010. Highway Capacity Manual. Transportation Research Board (TRB), Washington, DC, US. 1650 p.

Webster, F. V. 1958. Traffic Signal Settings. Road Research Lab Tech Papers No 39, UK, 1-44.

Wu, G.; Boriboonsomsin, K.; Zhang, L.; Barth, M. J. 2012. Simulation-based benefit evaluation of dynamic lane grouping strategies at isolated intersections, in 2012 15th International IEEE Conference on Intelligent Transportation Systems, 16-19 September 2012, Anchorage, AK, US, 1038-1043.

https://doi.org/10.1109/ITSC.2012.6338840

Yao, R.; Zhou, H.; Ge, Y.-E. 2018. Optimizing signal phase plan, green splits and lane length for isolated signalized intersections, Transport 33(2): 520-535.

https://doi.org/10.3846/16484142.2017.1297327 
Zakariya, A. Y.; Rabia, S. I. 2016. Estimating the minimum delay optimal cycle length based on a time-dependent delay formula, Alexandria Engineering Journal 55(3): 2509-2514.

https://doi.org/10.1016/j.aej.2016.07.029

Zhang, L.; Wu, G. 2012. Dynamic lane grouping at isolated intersections: problem formulation and performance analysis, Transportation Research Record: Journal of the Transportation Research Board 2311: 152-166.

https://doi.org/10.3141/2311-15

Zhao, J.; Ma, W.; Zhang, H. M.; Yang, X. 2013a. Increasing the capacity of signalized intersections with dynamic use of exit lanes for left-turn traffic, Transportation Research Record: Journal of the Transportation Research Board 2355: 49-59. https://doi.org/10.3141/2355-06

Zhao, J.; Ma, W.; Zhang, H. M.; Yang, X. 2013b. Two-step optimization model for dynamic lane assignment at isolated signalized intersections, Transportation Research Record: Journal of the Transportation Research Board 2355: 39-48. https://doi.org/10.3141/2355-05

Zhao, J.; Yao, J.; He, S.; Han, Y. 2017. Operational efficiency evaluation of intersections with dynamic lane assignment using field data, Journal of Advanced Transportation 2017: 2130385. https://doi.org/10.1155/2017/2130385

Zhong, Z.; Liu, H.; Ma, W.; Long, K. 2008. An optimization method of dynamic lane assignment at signalized intersection, in 2008 International Conference on Intelligent Computation Technology and Automation (ICICTA), 20-22 October 2008, Hunan, China, 1277-1280.

https://doi.org/10.1109/ICICTA.2008.264 